\title{
L'Asperger quotidiano - un piccolo esempio dalla Praxis
}

Alessia Schinardi

Corrispondenza:

Dr. med. Alessia Schinardi Kantonsspital Winterthur Kinderklinik SPZ

Brauerstrasse 15

CH-8401 Wintherthur

Tel. 0522663713

Fax 0522663509

alessia.schinardi@ksw.ch
I disturbi dello spettro autistico, caratterizzati da disturbi dell'interazione sociale, della comunicazione e da un ristretto campo di interessi, vengono diagnosticati sempre più spesso sia nel bambino che nell'adulto. Anche se attualmente sono più di moda i disturbi legati al deficit da attenzione e iperattività, fidatevi: presto diventeranno $i$ sintomi più diffusi nell'ambito della psicopatologia della vita quotidiana maschile. NESSUN MASCHIO verrà risparmiato.

Insomma, si era ripresa bene, con me non parla, falla parlare tu in Italiano... così forse si deciderà a riprendere l'antidepressivo..

Il solito pacco, i colleghi mi regalano i loro pazienti, ma non ne accettano nessuno in cambio... Certo che i pazienti sono più contenti quando parlano in madrelingua..

Infatti, la giovane donna mi sorride e ringrazia di esser entrata a far parte della mia corte di «clienti» ma non era il caso, lei non voleva venire, si risolverà. Mi spiega che il collega l'aveva aiutata a superare il mobbing nel vecchio posto di lavoro... omette di aver interrotto l'antidepressivo... poi non so' perché son ricaduta... non è che gli antidepressivi rendono dipendenti...

Neanche dopo che una li smette di propria iniziativa, come ha fatto lei.

Ma và meglio, sono contenta di parlare con lei, non credo che col suo collega avrei il coraggio di parlare di mio marito, è così gentile...

Chi?

Il suo collega intendo, non abbiamo mai parlato di mio marito, ma negli ultimi tempi mi ha fatto molto piangere...

Chi?

Adesso nel mentre che piange, mi racconti e risponda per favore alle mie domande senza preoccuparsi, noi psichiatri siamo famosi perché facciamo domande strane... vedo dalla cartella che suo marito è un informatico in una multinazionale...

Niente è successo, tutto come prima... solo che negli ultimi tempi devo piangere molto... ma và meglio...

Mi parli di cosa la affligge adesso.

Niente una cazzata, mi scusi, insomma, una Dummheit, mi sono arrabbiata con mio marito... perché ha fatto una scenata davanti ad amici che ci volevano subaffittare il loro appartamento.

???

Si è messo a gridare che lui non vuole traslocare, meglio morto, traslocherà solo definitivamente in un appartamento di proprietà... Gli amici non sapevano della sua allergia al cambiamento, ma non era il caso di reagire così... io volevo sotterrarmi! Insomma ha fatto un casino... scusi, ein Theater, genau, gli amici erano scioccati abbiamo cercato di cambiare argomento... ma io mi sono vergognata da morire, mi sono sentita come una donna musulmana che non ha diritto di parola...

Tu fai quello che vuoi... ha gridato, come se non fossi sua moglie, lui non ne vuol sentir parlare... invece era una buona proposta... io ci avrei fantasticato volentieri... è sbagliato?

Also, il solito stronzo egoista come molti altri... E poi?

Siamo andati via al più presto...

Vi siete chiariti?

E successo da pochi giorni, non li ho più sentiti, ma credo che i nostri amici non si faranno sentire per un po' $^{\prime}$....

No, dico, lei e suo marito ne avete riparlato?...

Per carità! Non c'è dialogo... non ho la forza di affrontarlo!... ma ho capito tante cose... vede siamo sempre soli e io ho imparato ad evitare certi argomenti...

Lui sta' bene?

E quello che dico a mia suocera: guardi come sta' bene! Anche se a sentir lui si lamenta sempre, non e' depresso, ma non gli và bene niente... e mi fa' depressa a me!

Insomma non si rende conto di come si sente lei, non è empatico, come dire, non riesce a immedesimarsi in lei...

Questo sarebbe veramente chiedere troppo! Io ho sempre pensato di essere come un libro aperto, almeno tutti mi dicono così, tutti tranne lui... può andare avanti per ore a lamentarsi senza notare che io covo pensieri suicidi...

Mi parli di lui, dei suoi interessi.

Sua madre dice sempre che la prima parola che ha detto è «auto». Ha una collezione dal 1990 della rivista «Auto sportive» e conosce a memoria i prezzi di tutte le auto nuove e usate, questo però l'ho scoperto però per caso... è normale?...

Legge altro?

A parte questa rivista e i suoi manuali ... no... però gli piace molto la TV.

Cosa?

Un po'di tutto, ma soprattutto lo zapping? ???

Cambia velocemente canale... senza fermarsi, io vado via... mi viene il mal di testa, credo che lo diverta... E' grave? 

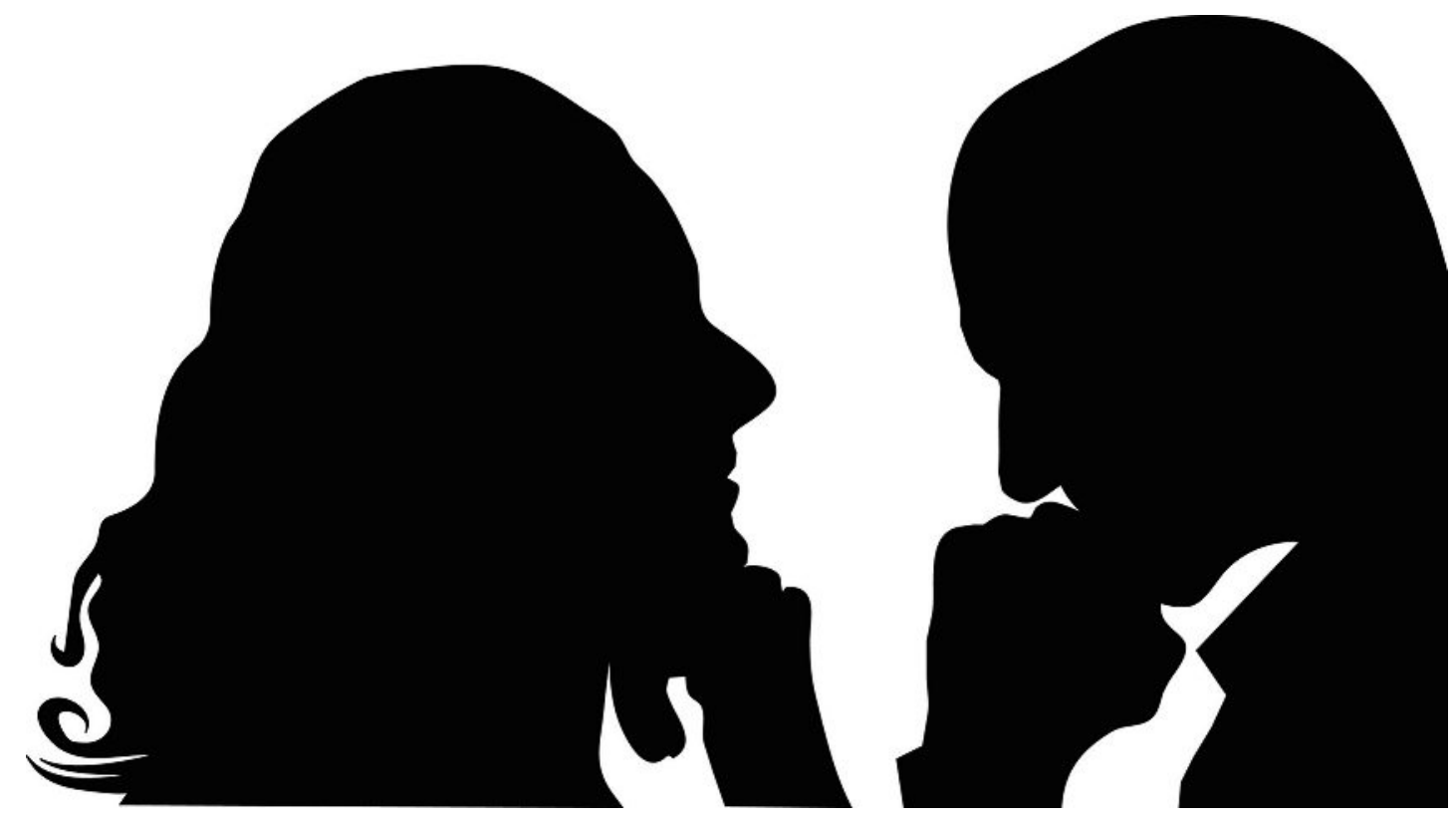

Mi dica se ho capito bene, nel mentre però si soffi il naso e beva un po' così può fare altre lacrime: suo marito è un manager di successo, ha pochi interessi ma in ambiti comuni. Vivete soli, lei si sente sola e incompresa mentre lui sembra contento così.

Esatto! Sono io che sto' male... ma forse non è tutta colpa mia...

Mentre il mio emisfero destro mostra empatia, il mio emisfero sinistro apre il cassetto dell'Asperger, gli autistici «high functioning»:

Non dica le parole colpa, giusto o sbagliato, normale, per favore, solo parole tabù per noi psichiatri!

Come?

Vada avanti, poi glielo spiego... Mi parli dei vostri amici, del tempo libero.

Una volta all'anno c'è una cena obbligatoria e allora vedo che i suoi colleghi lo stimano, anche se lo definiscono «lapidario». In queste occasioni mi sono accorta che anche se lui non parla mai di sé e del suo lavoro, con la scusa che è noioso parlare di programmazione industriale, i suoi colleghi lo fanno e rendono l'argomento interessante anche per chi non se ne intende come me... Mio marito dice che non vuol frequentare i colleghi di lavoro nel tempo libero e anche in questo, come in tutto, è irremovibile, anche se non ha tempo, non abbiamo il tempo di farci altri amici... non abbiamo amici in comune in realtà! Siam sempre soli.

Quindi non ha amici ma colleghi: Può condividere un piacere con gli altri? Per esempio la macchina nuova...

Macché devo raccontare io tutto ai suoceri! Pensi che non ha voluto portare l'iPhone in vacanza in Italia con la scusa che per le telefonate in Italia avrebbe dovuto comunque usare il vecchio cellulare. Ma non è che lo faccia perché non vuole ostentare, ho l'impressione che lui non vuole che gli altri tocchino le sue cose...

Sembra che... non voglio dire che non ha piacere nel condividere qualcosa, ma può prestare qualcosa?
Prestare, regalare e sorprese non sono parole che appartengono al suo vocabolario...

Suppongo che sia molto ordinato...

Solo per le sue cose... le sue cose sono solo sue e le conserva in un ordine religioso. A lungo ho pensato che fosse ossessivo! Le cose mie invece sono nostre e non gliene importa.

Mi dica, sa raccontare barzellette?

Come?

Gliel'ho detto che noi psichiatri facciamo domande strane: suo marito sa raccontare barzellette? Gli piacciono?

Veramente non mi ricordo in tutti questi anni, che ne abbia raccontato una: Lui ha difficoltà anche a fare giochi di società, le poche volte che capita lui sta' a guardare ... però ... Copia le mie frasi, mi ricordo che una volta mia cugina doveva assistere per lavoro ad un concerto dei Pooh... insomma, per consolarla le ho detto che i Pooh sono i nostri Rolling Stones...

!?!

Io non so' come la pensi lei sui Pooh, mio marito ha sentito, si è sganasciato dalle risate, lo ha ripetuto all'infinito e il giorno dopo mi ha detto: allora I Ricchi e Poveri sono i nostri Abba!

E lo ripeteva sempre... anche se lei non rideva più? Lei dice che non sa dire barzellette, ma si diverte se qualcuno le racconta...

Diciamo che quando lo fanno ridere sono grossolane... e adesso che ci penso trovo esagerato il modo in cui ride, molto rumoroso...con singhiozzi, irrispettoso quasi... ma forse è solo perché è cosi raro vederlo allegro, che mi sembra strano..

Facciamo un altro riassunto. Ho capito dal suo racconto, che suo marito ha scarse competenze sociali: non frequenta nessuno nel tempo libero, non parla di se o del suo lavoro, ha difficoltà a capire come si sente chi gli sta' di fronte, che non é una persona particolarmente flessibile, ma che questo gli è indifferente, lui è contento così... 
Sul fatto che lui non sia una persona flessibile, che si non si adatta, lo so' bene. Facciamo sempre ciò che vuole lui! Quando io voglio far qualcosa su cui lui non è d'accordo, non riesco a convincerlo, si mette ad urlare... lo devo mettere davanti al fatto compiuto e non mi perdona mai..

Per esempio?

Per esempio anni fa ho provato a convincerlo di andare a veder un Musical. Si è messo a gridare che lui odia i Musical, punto. Ho comprato due biglietti e gli ho detto che se non mi avrebbe accompagnato sarei andata con un'amica. Ha accettato subito naturalmente. Il Musical si è rivelato un fiasco e lui si è lamentato per mesi. Non prendo più iniziative.

Ecco parliamo di voi due, prenda un nuovo Kleenex, mi racconti come è nei suoi confronti? Siete sposati da 10 anni. La guarda negli occhi?

Sì, a me sì, ma per esempio, se io incontro un conoscente per strada lui si attacca improvvisamente alle vetrine, io all'inizio ci rimanevo male, lo volevo presentare, adesso non ci faccio più caso...

Ha difficoltà a parlare agli estranei, quindi.

Gira ore a vuoto, prima di chiedere la via! Al ristorante lascia che ordini io e guai se faccio un errore! Invece di parlare con il cameriere se la prende con me!

Mi faccia un esempio.

Eravamo in Francia e io ho ordinato per lui una bistecca ben cotta, lui mi ha fatto una scenata appena il cameriere si è allontanato anziché dire che la voleva al sangue.

Mi racconti ancora di cose bizzarre, che la fanno impazzire: Mi è caduto il ferro da stiro sul parquet, mi ha rimproverato per mesi, anche se era chiaro che non lo avevo fatto apposta... mi ha fatto sentire una bambina. Non voleva smettere...

Quando smette?

Quando me ne vado... vado spesso in cantina a lavare...

Non si accorge di come sta lei?

Può parlare per ore di ciò che lo interessa e non si accorge che sono annoiata... Altre volte invece mi si avvicina mi sbatte in faccia le cose che vuole mostrarmi, quasi sempre un'auto nuova... Mi piantona nel bagno, mi ci devo rinchiudere nel bagno. Alla fine glielo ho detto che non era il caso di farlo, all'inizio pensavo che fosse amore... il non potermi star lontano, poi mi son venute altre ipotesi in mente...

Per esempio?

Che è viziato, e vuole subito tutte le attenzioni Egocentrico dite voi, no? Lo so che mi ama anche se non me lo dice, ma é come se avesse difficoltà col fatto che siamo in due.

Also, lei si sforza di comprenderlo, ma lui non la capisce, neanche dopo tanti anni... non si sente compresa...

Compresa? A volte mi sento ET, un'extraterrestre. Mi racconti.

Per esempio lui vuole che io usi un linguaggio preciso, si attacca alle parole, secondo me per prendere tempo... una volta cucinavo gli spaghetti e volevo che mi avvicinasse lo scolapasta, invece ho detto «mi passi la pentola?» Mentre avevo la pentola bollente degli spaghetti in mano, tra di noi c'era solo lo scolapasta, lui non si è mosso, ha detto «quale pentola?». Io mi sono bruciata, volevi dire scolapasta... mi ha detto lui... ne ho altri di esempi del suo perfezionismo.

Può essere che suo marito abbia difficoltà a capire una situazione globalmente, dai gesti, dal tono e si basi soprattutto sul contenuto formale delle informazioni?

Come?

Manca la complicità, gesti e segni che avete fatto vostri nel corso del tempo.

Mi porge le cose da buttare anche se ho entrambe le mani occupate... é infantile vero?

Guardo l'ora, la cliente piange ancora, se non riesco a dirle qualcosa di costruttivo entro la fine della seduta piangerò anch'io: il mio Ego mi rinfaccerà tutti gli Ausbildungen sulle risorse... non ci sarà una prossima seduta, questa torna a casa e si spara...

Adesso le dico qualcosa di positivo su di lui: fa' la dichiarazione dei redditi da solo, sa riparare qualsiasi elettrodomestico in casa...

Come fa' a saperlo?

E le vuole molto bene, anche se non glielo ha mai detto.

Ma quanti anni ancora dovranno passare perché parli di noi al plurale? Prima che di sua iniziativa mi porti la borsa, mi versi da bere, che ordini per due al cameriere?

Adesso piango anch'io.

Mi scusi, dovrei a parlare di me e invece, lo so che non si possono cambiare gli assenti, me lo ha detto il suo collega, mi ha detto che anche lei è del compartimento...

Sì, signora sono anch'io ad orientamento cognitivo-comportamentale, ma la lingua batte dove il dente duole...e vorrei che si sfogasse...

Se le dicessi, generalizzando, che molti mariti sono come il suo si spara, se relativizzo coll'ampio e diffuso spettro fra normalità ed estremi esempi di mascolinità fino all'autismo mi spara a me...

Signora, se volesse divorziare sarebbe andata da un avvocato! Also, lei non si vuole liberare del suo problema. Io le posso mostrare diverse tecniche per ridimensionare il suo fardello fino ridurlo ad un volume sopportabile... come la sua borsa...

La mia borsa?

La sua borsa è in realtà una piccola valigia, anche se lei abita all'angolo. Io le posso insegnare a vedere il positivo del disfunzionale... e «trasformarlo» o viverlo in un modo per lei accettabile...

Per esempio?

Per esempio è puntuale, è affidabile, sta sempre con lei, non lo deve dividere con nessuno, non la tradirà mai... il solo pensiero di cambiare donna lo fa' star male! Ecco l'ho fatta ridere, ci vediamo la prossima volta.

Volete sapere le mie diagnosi? Non posso, son vincolata dal segreto professionale... 